\title{
SPACES OF FUNCTIONS WITH VALUES IN A BANACH ALGEBRA $\left({ }^{(}\right)$
}

\author{
BY \\ G. PHILIP JOHNSON( $\left.{ }^{2}\right)$
}

Introduction. In the following pages certain spaces of abstract-valued functions are examined. Throughout the paper $A$ will denote a commutative Banach algebra. In analogy to the group algebra $L^{1}(G)$ of a locally compact Abelian group $G$, that is, the space of absolutely integrable complex-valued functions on $G$, we form the set $B^{1}=B^{1}(G, A)$ of Bochner integrable functions defined to $A$ from $G$. $B^{1}$ is first of all a Banach space and it becomes a commutative Banach algebra if multiplication of two elements $f, g \in B^{1}$ is defined by the convolution formula, $(f * g)(x)=\int_{G} f(x y) g\left(y^{-1}\right) d y$.

For the theory of the Bochner integral we shall rely mainly on the presentation in Hille's book [2, pp. 35-49]. Although the development there uses the Lebesgue measure for finite dimensional Euclidean spaces, the theorems which we shall need hold as well for more general measure spaces, in particular for a locally compact group with Haar measure. The calculus for the generalized convolution carries over directly from that for numerical functions and will be assumed. We note that the convolution of a function in $B^{1}$ and a function in $L^{1}(G)$ is well defined and in $B^{1}$.

To the greatest possible extent the notation and definitions are those of Loomis [3]. "Maximal ideal" means regular maximal ideal throughout. Special conventions are as follows: $f=f(x), g=g(x), \cdots$ denote elements of $B^{1}$. For the most part complex-valued functions are assigned Greek letters regardless of the set on which they are defined. $\chi$ is always used for the characteristic function of a set and $\mu$ is the Haar measure on $G$. $\mathfrak{M}_{B}$ denotes the maximal ideal space of $B^{1}, \mathfrak{T l}_{A}$ that of $A$, and $\hat{G}$, the character group of $G$, is the maximal ideal space of $L^{1}$. Typical elements are $M_{B}, M$, and $\alpha$ respectively. Subscripts distinguish the various norms. For example, $\|f\|_{B}$ $=\int\|f(x)\|_{A} d x, f \in B^{1}$.

For any complex or $A$-valued function on $G$ and $x \in G$, the subscript $x$ applied to the function denotes its translate by $x$. The symbol ^ used with

Presented to the Society, April 13, 1956 under the title of Spaces of functions with values in a normed ring; received by the editors May 7, 1957 and, in revised form, February 2, 1958.

${ }^{1}$ Many of the results in the first three sections were obtained independently by Alvin Hausner and were presented at a different meeting of the Society at the same time as the author presented his results (see Bull. Amer. Math. Soc. vol. 62 (1956) pp. 383-384).

2 This paper is based on a doctoral thesis submitted to the Graduate Faculty of the University of Minnesota in the summer of 1956. The author is much indebted to Professor $B$. R. Gelbaum who suggested the topic and gave unstintingly of his time in guiding preparation of the thesis. 
an element of a Banach algebra denotes its abstract Fourier transform and $E^{\prime}$ is the complement of the set $E$.

The principal tool in this development is the Gelfand representation and there is a consequent preoccupation with the maximal ideals of $B^{1}$. In the first section some of the $L^{1}$ results connecting translation invariance and ideals are shown to hold in $B^{1}$. An analogue of the theorem stating that a convolution is in the span of translates of either of its arguments is proved. (This theorem has been proved for almost periodic functions by von Neumann [4, p. 457] and its statement for elements of $L^{1}$ is given by Segal [6, p. 94].) These results are applied in the second section in an analysis of the structure of $\mathfrak{M}_{B}$. It is found that a maximal ideal of $B^{1}$ is an ordered pair $(\alpha, M)$, $\alpha \in \hat{G}, M \in \mathfrak{M}_{A}$; and, in fact, $\mathfrak{M}_{B}$ is homeomorphic to $\hat{G} \times \mathfrak{M}_{A}$ with the weak topologies. The connection between the pairs $(\alpha, M)$ and the $M_{B}$ is a generalized integral formula for the Fourier transform. In the third section it is shown that if $A$ is a group algebra, $B^{1}$ is (isometric and isomorphic to) a group algebra. The fourth section is concerned with an isometric isomorphism $T$ of $B^{1}(G, A)$ onto a like algebra $B^{1}(\tilde{G}, \tilde{A})$. We obtain conditions under which $T$ gives rise to isomorphisms of $G$ onto $\widetilde{G}$ and of $A$ onto $\tilde{A}$ in terms of which $T$ can be expressed in a particularly simple fashion. An example shows that in general neither $G$ and $\widetilde{G}$ nor $A$ and $\widetilde{A}$ need be isomorphic.

1. Approximation of convolution and translation invariance in ideals. Let $f$ be a continuous $A$-valued function with compact carrier $C$ and let $V$ be a measurable open neighborhood of the identity $e$. There exists a finite set $\left\{a_{i} V\right\}, i=1,2, \cdots, n$, of translates of $V$ which cover $C$. Let $E_{1}=C \cap a_{1} V$, $E_{2}=C \cap a_{2} V \cap E_{1}^{\prime}, \cdots, E_{n}=C \cap a_{n} V \cap\left(\cup_{i=1}^{n-1} E_{i}\right)^{\prime}$. These are disjoint measurable sets whose union is $C$ and for each $i, E_{i} \subset a_{i} V$. Form a simple function $f_{V}=\sum_{i} f\left(x_{i}\right) \chi_{E_{i}}$, where $x_{i}$ is arbitrary in $E_{i}$. (If $E_{i}$ is empty, the choice of $x_{i}$ is immaterial.)

Lemma 1.1. If $f$ is continuous with compact carrier $C$ and $\epsilon>0$, there exists a measurable open neighborhood $V_{\epsilon}$ of e such that for any measurable open neighborhood $V$ of e contained in $V_{\mathrm{e}}$ and corresponding $f_{V}$,

$$
\left\|\int f(x) d x-\int f_{V}(x) d x\right\|_{A}=\left\|\int f(x) d x-\sum_{i} f\left(x_{i}\right) \mu\left(E_{i}\right)\right\|_{A}<\epsilon .
$$

Proof. If $f \neq 0 \in B^{1}$, there exists, by the uniform continuity of $f$, a neighborhood $W$ of $e$ such that $x y^{-1} \in W$ implies $\|f(x)-f(y)\|_{A}<\epsilon / \mu(C)$. Choose $V_{\epsilon}$ such that $V_{\epsilon} V_{\epsilon}^{-1} \subset W$. If $x \in C$, then $x \in E_{i}$ for some $i$, whence $x x_{i}^{-1} \in V V^{-1}$ $\subset W$. Therefore

$$
\left\|\int f(x) d x-\int f_{V}(x) d x\right\|_{A} \leqq \int\left\|f(x)-f_{V}(x)\right\|_{A} d x<\epsilon .
$$

The following lemma contains the essentials of the convolution approxima- 
tion referred to in the introduction. The lemma will also be used in the second section.

LEMma 1.2. Let $V_{0}$ be a measurable open neighborhood of $e$ and let $\epsilon>0$ be given. If $f \in B^{1}$ and $g$ is a continuous $A$-valued function with compact carrier $C_{g}$, then there exists a measurable open neighborhood $V$ of $e$ contained in $V_{0}$ such that for a measurable subdivision $\left\{E_{i}\right\}$ of $C_{g}$ obtained as above from $V$ and $y_{i} \in E_{i}, i=1, \cdots, L$,

$$
\left\|f * g-\sum_{i} f_{y_{i}^{-1}} g\left(y_{i}\right) \mu\left(E_{i}\right)\right\|_{B}<\epsilon .
$$

Proof. We assume that neither $f$ nor $g$ is the zero element of $B^{1}$, for otherwise the assertion is trivial.

CASE 1. $f$ is continuous with compact carrier $C_{f}$. Then $f * g$ is continuous and

$$
(f * g)(x)=\int_{C_{o}} f\left(x y^{-1}\right) g(y) d y .
$$

If $y \in C_{\theta}$ and $x \notin C_{\theta} C_{f}$, then $x y^{-1} \notin C_{f}$, so that $f * g$ vanishes outside the compact set $C=C_{g} C_{f}$.

Let $\left\{E_{i}\right\}$ be any finite collection of disjoint measurable sets whose union is $C_{g}$ and take $y_{i} \in E_{i}$. By virtue of the uniform continuity of $f$ and $f * g$, one can find a neighborhood $U$ of $e$ such that $x_{1} x_{2}^{-1} \in U$ implies

$$
\begin{aligned}
\| \sum_{i}\left[f\left(x_{1} y_{i}^{-1}\right)\right. & \left.-f\left(x_{2} y_{i}^{-1}\right)\right] g\left(y_{i}\right) \mu\left(E_{i}\right) \|_{A} \\
& \leqq \sum_{i}\left\|f\left(x_{1} y_{i}^{-1}\right)-f\left(x_{2} y_{i}^{-1}\right)\right\| A\left\|g\left(y_{i}\right)\right\|_{A} \mu\left(E_{i}\right)<\epsilon / 3 \mu(C)
\end{aligned}
$$

and

$$
\left\|(f * g)\left(x_{1}\right)-(f * g)\left(x_{2}\right)\right\|_{A}<\epsilon / 3 \mu(C) .
$$

Let $U_{0}$ be a measurable open neighborhood of $e$ such that $U_{0} U_{0}^{-1} \subset U$. As before, construct from the translates of $U_{0}$ a finite collection $\left\{W_{n}\right\}$, $n=1, \cdots, N$, of disjoint measurable sets whose union is $C$. Pick $x_{n} \in W_{n}$. Since for each $n, f\left(x_{n} y^{-1}\right) g(y)$ is continuous and vanishes outside $C_{\theta}$, there exists by the preceding lemma a neighborhood $V_{n}$ of $e$ such that

$$
\left\|\int f\left(x_{n} y^{-1}\right) g(y) d y-\sum_{i} f\left[x_{n}\left(y_{i}^{(n)}\right)^{-1}\right] g\left(y_{i}^{(n)}\right) \mu\left(E_{i}^{(n)}\right)\right\|_{A}<\epsilon / 3 \mu(C)
$$

where $\left\{E_{i}^{(n)}\right\}$ is a subdivision of $C_{0}$ provided by $V_{n}$ and $y_{i}^{(n)} \in E_{i}^{(n)}, i=1, \cdots$, $L_{n}$. Let $V=\left(\cap_{n} V_{n}\right) \cap V_{0}$. Then $V \subset V_{0}$ and for $\left\{E_{i}\right\}$, a subdivision of $C_{0}$ given by $V, y_{i} \in E_{i}, i=1, \cdots, L$, and for every $n$, 


$$
\left\|(f * g)\left(x_{n}\right)-h\left(x_{n}\right)\right\|_{-1}<\epsilon / 3 \mu(C), \quad \text { where } \quad h=\sum_{i} f_{y_{i}^{-1}} g\left(y_{i}\right) \mu\left(E_{i}\right) .
$$

Now if $x \in C$, there exists an $n$ such that $x \in W_{n}$. Since $W_{n}$ is contained in a translate of $U_{0}, x x_{n}^{-1} \in U_{0} U_{0}^{-1} \subset U$. Hence

$$
\begin{array}{r}
\|(f * g)(x)-h(x)\|_{A} \leqq \\
+\left\|(f * g)(x)-(f * g)\left(x_{n}\right)\right\|_{A}+\left\|(f * g)\left(x_{n}\right)-h\left(x_{n}\right)\right\|_{A} \\
+\left\|\sum_{i}\left[f\left(x_{n} y_{i}^{-1}\right)-f\left(x y_{i}^{-1}\right)\right] g\left(y_{i}\right) \mu\left(E_{i}\right)\right\|_{A}<\epsilon / \mu(C)
\end{array}
$$

for all $x \in C$. Since for every $i, f_{y_{i}-1}$ vanishes outside $C$,

$$
\|f * g-h\|_{B}<\mu(C) \cdot \epsilon / \mu(C)=\epsilon .
$$

CASE 2. $f$ is arbitrary in $B^{1}$. It is possible to select a continuous function $f^{(1)}$ with compact carrier and from some $V \subset V_{0}$ to construct, as in Case 1 , a function $h^{(1)}=\sum_{i} f_{y_{i}}^{(1)} g\left(y_{i}\right) \mu\left(E_{i}\right)$ such that $\left\|f-f^{(1)}\right\|_{B}$ and $\left\|f^{(1)} * g-h^{(1)}\right\|_{B}$ are arbitrarily small. Let $h$ be the function obtained from $h^{(1)}$ by replacing $f^{(1)}$ by $f$. Then since $g$ is bounded, $\left\|h-h^{(1)}\right\|_{B}$ and, consequently, $\|f * g-h\|_{B}$ are small.

THEOREM 1.1. If $f, g \in B^{1}$ and $\epsilon>0$, there exists a finite sum $h$ of $A$ multiples of translates of $f$ such that $\|f * g-h\|_{B}<\epsilon$.

Proof. For some continuous function $g^{(1)}$ with compact carrier and, by Lemma 1.2, for a finite sum $h$ of $A$ multiples of translates of $f,\left\|g-g^{(1)}\right\|_{B}$ and $\left\|f * g^{(1)}-h\right\|_{B}$ are small, whence $\|f * g-h\|_{B}$ is small.

TheOREM 1.2. Any closed translation invariant subspace of $B^{1}$ which admits multiplication by elements of $A$ is an ideal.

Proof. Let $I$ be the subspace and take $f \in I, g \in B^{1}$. By Theorem 1.1, $f * g$ is the limit of sums of $A$ multiples of translates of $f$. Each of these is in $I$ and therefore, since $I$ is closed, $f * g \in I$.

The converse of this theorem holds if the ideal is closed and regular. We shall use an approximate identity in the proof.

Definition. A directed system $\{u\}$ of functions in $L^{1}$ is called an approximate identity for $B^{1}$ in case $\lim _{u}\|u * f-f\|_{B}=0$ for every $f \in B^{1}$.

The following theorem, a paraphrase of Theorem 3.7.1 of [2], asserts that approximate identities for $B^{1}$ exist.

Theorem 1.3. Let $\epsilon>0$ be given. Corresponding to $f \in B^{1}$ there exists a neighborhood $V$ of the identity such that if $u$ is any non-negative function of $L^{1}$ vanishing outside $V$ and $\int u d x=1$, then $\|u * f-f\|_{B}<\epsilon$.

Theorem 1.4. $A$ regular ideal in $B^{1}$ is translation invariant. $A$ closed ideal admits multiplication by elements of $A$.

Proof. Let $I$ be a regular ideal in $B^{1}$ and $u$ an identity modulo $I$. Under 
the homomorphism of $B^{1}$ induced by $I, u$ is carried into the identity $\bar{u}$ of $B^{1} / I$. Let $f \in I$. Since $f * u_{x}=f_{x} * u$, we have in $B^{1} / I, 0=\bar{f} \bar{u}_{x}=\bar{f}_{x} \bar{u}=\bar{f}_{x}$. Hence $f_{x} \in I$.

Suppose $I$ is a closed ideal. For $a \in A$ and each $u \in\{u\}$, an approximate identity, $u * a f=a u * f \in I$, and $\lim _{u} u * a f=a f$. Since $I$ is closed, $a f \in I$.

2. The maximal ideals of $B^{1}$. The fact that each pair $(\alpha, M), \alpha \in \hat{G}$, $M \in \mathfrak{N}_{A}$, gives rise to a maximal ideal of $B^{1}$ is easily arrived at, and indeed follows immediately from the next result.

THEOREM 2.1. For any character $\alpha \in \hat{G}$ the mapping $f \rightarrow \int f(x) \bar{\alpha}(x) d x$ is a continuous homomorphism of $B^{1}$ onto $A$.

Proof. The mapping is obviously linear and we have by the Fubini theorem

$$
\begin{aligned}
f * g & \rightarrow \iint f(x y) g\left(y^{-1}\right) d y \bar{\alpha}(x) d x=\iint f(x y) \bar{\alpha}(x y) d x g\left(y^{-1}\right) \bar{\alpha}\left(y^{-1}\right) d y \\
& =\int f(x) \bar{\alpha}(x) d x \int g(y) \bar{\alpha}(y) d y .
\end{aligned}
$$

Since $\left\|\int f \bar{\alpha} d x\right\|_{A} \leqq\|f\|_{B}$, the homomorphism is continuous. To show that it maps $B^{1}$ onto $A$ we take $\lambda \in L^{1}$ such that $\lambda(\alpha)=1$. If $a \in A$, we have $a \lambda \in B^{1}$ and $a \lambda \rightarrow a \int \lambda(x) \bar{\alpha}(x) d x=a \hat{\lambda}(\alpha)=a$.

Thus if $M$ is any maximal ideal of $A$, the product homomorphism $f \rightarrow \int f(x) \bar{\alpha}(x) d x \rightarrow\left(\int f(x) \bar{\alpha}(x) d x\right)^{\wedge}(M)$ is a homomorphism of $B^{1}$ onto the complex numbers. It follows that to each pair $(\alpha, M)$ there corresponds a maximal ideal $M_{B}^{(\alpha, M)}$ of $B^{1}$ where

$$
\hat{f}\left(M_{B}^{(\alpha, M)}\right)=\left(\int f(x) \bar{\alpha}(x) d x\right) \hat{(M)}
$$

for every $f \in B^{1}$. Because the homomorphism given by $M$ is a bounded linear transformation on $A$,

$$
\hat{f}\left(M_{B}^{(\alpha, M)}\right)=\int f(x)^{\wedge}(M) \bar{\alpha}(x) d x, \quad f \in B^{\prime} .
$$

THEOREM 2.2. If $\left(\alpha_{1}, M_{1}\right)$ is distinct from $\left(\alpha_{2}, M_{2}\right)$, then the corresponding maximal ideals of $B^{1}$ are distinct.

Proof. If $M_{1} \neq M_{2}$, take $a \in M_{1} \cap M_{2}^{\prime}$ and $\lambda \in L^{1}$ such that $\lambda\left(\alpha_{2}\right) \neq 0$. Then

$$
(a \lambda)^{\wedge}\left(M_{B}^{\left(\alpha_{1}, M_{1}\right)}\right)=\hat{\lambda}\left(\alpha_{1}\right) \hat{a}\left(M_{1}\right)=0 \neq \hat{\lambda}\left(\alpha_{2}\right) \hat{a}\left(M_{2}\right)=(a \lambda)^{\wedge}\left(M_{B}^{\left(\alpha_{2}, M_{2}\right)}\right) .
$$

If $\alpha_{1} \neq \alpha_{2}$, for $a \notin M_{2}$ and $\lambda \in L^{1}$ such that $\hat{\lambda}\left(\alpha_{1}\right)=0 ; \hat{\lambda}\left(\alpha_{2}\right) \neq 0$,

$$
(a \lambda)^{\wedge}\left(M_{B}^{\left(\alpha_{1}, M_{1}\right)}\right)=0 \neq(a \lambda)^{\wedge}\left(M_{B}^{\left(\alpha_{2}, M_{2}\right)}\right) .
$$


The integral formula $\left({ }^{*}\right)$ thus defines a one-to-one correspondence between $\hat{G} \times \mathfrak{M}_{A}$ and a subset of $\mathfrak{M}_{B}$. We have yet to prove that all $\mathfrak{M}_{B}$ is accounted for in this way, or equivalently, that for each $M_{B} \in \mathfrak{M}_{B}$, a character and a maximal ideal of $A$ can be found which are related to $M_{B}$ through the integral formula. The generalization of a well-known result due to Raikov [5] provides a candidate for the character.

THeOREM 2.3. For any $M_{B} \in \mathfrak{M}_{B}$ and $f \in B^{1} \cap M_{B}^{\prime}$, the function $\alpha_{B}$ defined by $\alpha_{B}(x)=\hat{f}_{x}\left(M_{B}\right) / \hat{f}\left(M_{B}\right)$ is independent of $f$ and is a character of $G$.

The proof in $[3$, p. 135] applies virtually unchanged.

If $A$ has an identity $e$, then $\lambda e \in B^{1}$ whenever $\lambda \in L^{1}$. In fact, if we assume, as we may, that $\|e\|_{A}=1$, then $L^{1}$ is isometric and isomorphic to the subalgebra $L^{1} e$ of $B^{1}$. Since this feature considerably facilitates working in $B^{1}$, several of the next results assume the presence of $e$ in $A$.

THEOREM 2.4. If $A$ has an identity and $M_{B} \in \mathbb{M}_{B}$, then $M_{B} \cap L^{1} \mathcal{C}$ is a maximal ideal of $L^{1} e$ and $\left\{\lambda: \lambda e \in M_{B} \cap L^{1} e\right\}$ is the maximal ideal of $L^{1}$ corresponding to $\alpha_{B}$.

Proof. Let $\{u\}$ be an approximate identity. Since not every element $u e$ can be in $M_{B}, M_{B} \cap L^{1} e \neq L^{1} e$ and hence is a maximal ideal.

Let $\alpha$ be the character corresponding to $\left\{\lambda: \lambda e \in M_{B} \cap L^{1} e\right\}$. If $\nu \in L^{1}$ is such that $\hat{\nu}(\alpha)=1$, clearly $\nu e$ is an identity modulo $M_{B} \cap L^{1} e$. Since $\nu_{x}=\hat{\nu}_{x}(\alpha) \nu$ $+\lambda_{0}$, where $\lambda_{0}(\alpha)=0,\left(\nu_{x} e\right)^{\wedge}\left(M_{B}\right)=\left(\nu_{x} e\right)^{\wedge}\left(M_{B} \cap L^{1} e\right)=\hat{\nu}_{x}(\alpha)$. Therefore

$$
\alpha_{B}(x)=\left(\nu_{x} e\right)^{\wedge}\left(M_{B}\right) /(\nu e)^{\wedge}\left(M_{B}\right)=\hat{\nu}_{x}(\alpha) / \hat{\nu}(\alpha)=\alpha(x)
$$

for every $x \in G$.

TheOREM 2.5. If $A$ has an identity and if $\lambda e \in L^{\prime} e$ is an identity modulo $M_{B} \in \mathfrak{M}_{B}$, then the mapping $a \rightarrow(a \lambda)^{\wedge}\left(M_{B}\right)$ is a homomorphism of $A$ onto the complex numbers. It is independent of $\lambda$, and the corresponding maximal ideal $M^{(B)} \in \mathscr{M}_{A}$ is such that for every $f \in B^{1}$

$$
\hat{f}\left(M_{B}\right)=\int f(x)^{\wedge}\left(M^{(B)}\right) \bar{\alpha}_{B}(x) d x .
$$

Proof. If a different identity $\nu e$ modulo $M_{B}$ is used, then $\lambda e-\nu e \in M_{B}$ and by Theorem 1.4, $a \lambda-a \nu \in M_{B}$ for every $a \in A$. Hence the mapping is independent of $\lambda$. In particular, since $(\lambda * \lambda e)^{\wedge}\left(M_{B}\right)=\left[(\lambda e)^{\wedge}\left(M_{B}\right)\right]^{2}=1,(a \lambda)^{\wedge}\left(M_{B}\right)$ $=(a \lambda * \lambda)^{\wedge}\left(M_{B}\right)$. Let $\bar{a}=(a \lambda)^{\wedge}\left(M_{B}\right) . a \rightarrow \bar{a}$ is linear and for $a_{1}, a_{2} \in A$

$$
\begin{aligned}
\bar{a}_{1} \bar{a}_{2} & =\left(a_{1} \lambda\right)^{\wedge}\left(M_{B}\right)\left(a_{2} \lambda\right)^{\wedge}\left(M_{B}\right)=\left(a_{1} a_{2} \lambda * \lambda\right)^{\wedge}\left(M_{B}\right) \\
& =\left(a_{1} a_{2} \lambda\right)^{\wedge}\left(M_{B}\right)=\left(a_{1} a_{2}\right)^{-} .
\end{aligned}
$$

$a \rightarrow \bar{a}$ is therefore a homomorphism of $A$ on to the complexes.

Now let $E$ be a set of finite measure in $G$ and let $f=a \chi_{E}, a \in A$. Since 
$\chi_{E} \in L^{1}$, we may write $\chi_{E}=\hat{\chi}_{E}\left(\alpha_{B}\right) \lambda+\lambda_{0}$, where $\hat{\lambda}_{0}\left(\alpha_{B}\right)=0$. Then $f(x)=\hat{\chi}_{E}\left(\alpha_{B}\right) a \lambda$ $+a \lambda_{0}$, and $\int f(x)^{\wedge}\left(M^{(B)}\right) \bar{\alpha}_{B}(x) d x=\hat{\chi}_{E}\left(\alpha_{B}\right) \hat{a}\left(M^{(B)}\right)$. On the other hand, since $a \lambda_{0} \in M_{B}, \hat{f}\left(M_{B}\right)=\hat{\chi}_{E}\left(\alpha_{B}\right)(a \lambda)^{\wedge}\left(M_{B}\right)=\hat{\chi}_{E}\left(\alpha_{B}\right) \hat{a}\left(M^{(B)}\right)$. Hence $\hat{f}\left(M_{B}\right)$ $=\int f(x)^{\wedge}\left(M^{(B)}\right) \bar{\alpha}_{B}(x) d x$. The integral formula has thus been proved for functions of the form $a \chi_{E}$. Since such functions constitute a fundamental set of $B^{1}$ and since the left and right hand sides of the formula are each linear functionals on $B^{1}$ which are equal on this set, they are equal throughout $B^{1}$. This completes the proof.

If $A$ does not have an identity, it can be embedded in a Banach algebra $A_{e}=\{a+c e: a \in A, c$ complex $\}$ which does. Let $B^{1}(e)=B^{1}\left(G, A_{e}\right)$. An element of $B^{1}(e)$ is the sum of an $A$-valued function and a complex-valued function multiplied by $e$. If $f+\lambda e$ is one such, then

$$
\|f+\lambda e\|_{B(e)}=\int\|f+\lambda e\|_{A_{e}} d x=\|f\|_{B}+\|\lambda\|_{1} .
$$

Thus $B^{1}(e)$ consists of the functions $f+\lambda e$, where $f \in B^{1}, \lambda \in L^{1}$. We note that $B^{1}$ is a closed ideal of $B^{1}(e)$. This follows from Theorem 1.2 ; for $B^{1}$ is a translation invariant subspace which admits multiplication by elements of $A_{e}$, and it is closed because it is complete with respect to the $B^{1}(e)$ norm.

Lemma 2.1. If $f \in B^{1}, \lambda \in L^{1}$, and $M_{B} \in \Re_{B}$, then $(\lambda * f) \wedge\left(M_{B}\right)=\hat{\lambda}\left(\alpha_{B}\right) \hat{f}\left(M_{B}\right)$.

Proof. Since $M_{B}$ is a closed subspace of $B^{1}$, it is a closed subspace of $B^{1}(e)$. By Theorem 1.4, it is translation invariant and admits multiplication by elements of $A_{e}$. Hence by Theorem $1.2, M_{B}$ is an ideal of $B^{1}(e)$. Thus if $f \in M_{B}, \quad \lambda * f=\lambda e * f \in M_{B}$. Therefore $(\lambda * f)^{\wedge}\left(M_{B}\right)=0=\hat{\lambda}\left(\alpha_{B}\right) \hat{f}\left(M_{B}\right)$, which proves the formula for this case.

If $f \notin M_{B}$, then $\alpha_{B}(x)=\hat{f}_{x}\left(M_{B}\right) / \hat{f}\left(M_{B}\right)$. Suppose $\lambda$ is a continuous function with compact carrier $C$ and let $\epsilon>0$ be given. Since Lemma 1.1 applies to $\lambda \bar{\alpha}_{B}$, there exists an open measurable neighborhood $V_{\epsilon}$ such that

$$
\left|\int \lambda(y) \bar{\alpha}_{B}(y) d y-\sum_{i} \lambda\left(y_{i}\right) \bar{\alpha}_{B}\left(y_{i}\right) \mu\left(E_{i}\right)\right|<\epsilon / 2,
$$

where $\left\{E_{i}\right\}$ is a subdivision of $C$ obtained from $V_{\epsilon}$ and $y_{i} \in E_{i}, i=1, \cdots, n$. Because $\lambda e$ is a continuous $A_{e}$-valued function with compact carrier, there exists, by Lemma 1.2, a neighborhood $V \subset V_{\epsilon}$ such that for a subdivision $\left\{E_{j}\right\}$ obtained from $V$ and $y_{j} \in E_{j}, j=1, \cdots, L$,

$$
\left\|\lambda * f-\sum_{j} f_{y_{j}^{-1}} \lambda\left(y_{j}\right) \mu\left(E_{j}\right)\right\|_{B}<\frac{\epsilon}{2}\left|\hat{f}\left(M_{B}\right)\right| .
$$

Therefore

$$
\left|\frac{(\lambda * f)^{\wedge}\left(M_{B}\right)}{\hat{f}\left(M_{B}\right)}-\sum_{j} \bar{\alpha}_{B}\left(y_{j}\right) \lambda\left(y_{j}\right) \mu\left(F_{i j}\right)\right|<\frac{\epsilon}{2} .
$$


Since $V \subset V_{\epsilon}$,

$$
\left|\frac{(\lambda * f)^{\wedge}\left(M_{B}\right)}{\hat{f}\left(M_{B}\right)}-\int \lambda(y) \bar{\alpha}_{B}(y) d y\right|<\epsilon .
$$

Thus $(\lambda * f)^{\wedge}\left(M_{B}\right)=\hat{\lambda}\left(\alpha_{B}\right) \hat{f}\left(M_{B}\right)$.

The two sides of the formula are continuous functions of $\lambda$ and are equal on a dense subset of $L^{1}$. Therefore $(\lambda * f)^{\wedge}\left(M_{B}\right)=\hat{\lambda}\left(\alpha_{B}\right) \hat{f}\left(M_{B}\right)$ for every $\lambda \in L^{1}$.

For $M_{B} \in \mathscr{M}_{B}$ the linear mapping $f+\lambda e \rightarrow \hat{f}\left(M_{B}\right)+\hat{\lambda}\left(\alpha_{B}\right)$ is also multiplicative, for by the lemma

$$
\begin{aligned}
(f+\lambda e) *(g+\nu e) & \rightarrow(f * g+\lambda * g+\nu * f)^{\wedge}\left(M_{B}\right)+(\lambda * \nu)^{\wedge}\left(\alpha_{B}\right) \\
& =\left[\hat{f}\left(M_{B}\right)+\lambda\left(\alpha_{B}\right)\right]\left[\hat{g}\left(M_{B}\right)+\hat{\nu}\left(\alpha_{B}\right)\right] .
\end{aligned}
$$

The mapping is therefore a homomorphism of $B^{1}(e)$ onto the complex numbers. Let $M_{B(e)}$ be the associated maximal ideal. Since $M_{B(e)} \cap L^{1} e$ $=\left\{\lambda e: \hat{\lambda}\left(\alpha_{B}\right)=0\right\}, \alpha_{B(e)}=\alpha_{B}$ (see Theorem 2.4). Therefore, by Theorem 2.5, there exists a maximal ideal $M^{(B(e))}$ of $A_{e}$ such that

$$
\begin{aligned}
(f+\lambda e)^{\wedge}\left(M_{B(e)}\right) & =\int[f(x)+\lambda(x) e]^{\wedge}\left(M^{(B(e))}\right) \bar{\alpha}_{B}(x) d x \\
& =\int f(x)^{\wedge}\left(M^{(B)}\right) \bar{\alpha}_{B}(x) d x+\hat{\lambda}\left(\alpha_{B}\right),
\end{aligned}
$$

where $M^{(B)}=M^{(B(e))} \cap A$. Note that $M^{(B)}$ cannot be $A$; for if it were, all $B^{1}$ would be mapped by $M_{B}$ into zero. Hence $M^{(B)}$ is a maximal ideal of $A$ and $\hat{f}\left(M_{B}\right)=\int f(x)^{\wedge}\left(M^{(B)}\right) \bar{\alpha}_{B}(x) d x$.

The following characterization of $\mathfrak{M}_{B}$ has been completely demonstrated.

Theorem 2.6. The mapping $(\alpha, M) \rightarrow M_{B}^{(\alpha, M)}$ is a one-to-one mapping of $\hat{G} \times \mathbb{M}_{A}$ onto $\mathfrak{M}_{B}$, and

$$
\hat{f}\left(M_{B}^{(\alpha, M)}\right)=\int f(x)^{\wedge}(M) \bar{\alpha}(x) d x
$$

for all $f \in B^{1}$.

The remainder of this section is given to showing that in the sense of the weak topology the correspondence between $\hat{G} \times \Re_{A}$ and $\mathfrak{M}_{B}$ is a homeomorphism.

Lemma 2.2. Let $U \subset \hat{G}$ be open with respect to the weak topology of $\hat{G}$. Then $\left\{M_{B}: \alpha_{B} \in U\right\}$ is open in the weak topology of $\mathfrak{M}_{B}$.

Proof. Let $M_{B}^{(0)}=\left(\alpha_{0}, M_{0}\right)$ be an arbitrary element in $\left\{M_{B}: \alpha_{B} \in U\right\}$. It suffices to show that there exists a neighborhood $N$ of $M_{B}^{(0)}$ such that $N^{T} \subset\left\{M_{B}: \alpha_{B} \in U_{1}\right\}$, where $U_{1}=\left\{\alpha:\left|\lambda(\alpha)-\lambda\left(\alpha_{0}\right)\right|<\epsilon\right\}, \lambda \in L^{1}$. Therefore pick 
$f \in B^{1}$ such that $\left|\hat{f}\left(M_{B}^{(0)}\right)\right|>1$ and let

$$
N_{1}=\left\{M_{B}:\left|\hat{f}\left(M_{B}\right)\right|>1\right\} .
$$

$N_{1}$ is then an open neighborhood of $M_{B}^{(0)}$ as are

$$
N_{2}=\left\{M_{B}:\left|(\lambda * f)^{\wedge}\left(M_{B}\right)-(\lambda * f)^{\wedge}\left(M_{B}^{(0)}\right)\right|<\epsilon / 2\right\},
$$

and

$$
N_{3}=\left\{M_{B}:\left|\hat{f}\left(M_{B}\right)-\hat{f}\left(M_{B}^{(0)}\right)\right|<\epsilon / 2 \max \left(\left|\hat{\lambda}\left(\alpha_{0}\right)\right|, 1\right)\right\} .
$$

By Lemma 2.1, for all $M_{B} \in N=N_{1} \cap N_{2} \cap N_{3}$

$$
\begin{aligned}
\mid \hat{\lambda}\left(\alpha_{B}\right)- & \lambda\left(\alpha_{0}\right)|<| \hat{\lambda}\left(\alpha_{B}\right) \hat{f}\left(M_{B}\right)-\hat{\lambda}\left(\alpha_{0}\right) \hat{f}\left(M_{B}\right) \mid \\
& \leqq\left|(\lambda * f)^{\wedge}\left(M_{B}\right)-(\lambda * f)^{\wedge}\left(M_{B}^{(0)}\right)\right|+\left|\hat{x}\left(\alpha_{0}\right)\right|\left|\hat{f}\left(M_{B}^{(0)}\right)-\hat{f}\left(M_{B}\right)\right| \\
& <\epsilon .
\end{aligned}
$$

Lemma 2.3. If $f \in B^{1}$, then $\int f(x) \bar{\alpha}(x) d x$ is in the weak topology a uniformly continuous $A$-valued function over $\hat{G}$.

The proof is omitted since the proof in $[3,34 \mathrm{C}]$ can be applied readily.

TheOREM 2.7. With the weak topologies on $\hat{G}, \mathfrak{M}_{A}$, and $\mathfrak{M}_{B}, \hat{G} \times \mathfrak{T}_{A}$ and $\mathfrak{M}_{B}$ are homeomorphic.

Proof. We first choose a $\hat{G} \times \mathbb{M}_{A}$ sub-basis element $N\left(\alpha_{0}, M_{0}\right)$ about a point $\left(\alpha_{0}, M_{0}\right)$ and find an $\mathfrak{T}_{B}$-neighborhood of $M_{B}^{(0)}=\left(\alpha_{0}, M_{0}\right)$ contained in it. For $\lambda \in L^{1}, a \in A$, let

$$
N\left(\alpha_{0}, M_{0}\right)=\left\{(\alpha, M):\left|\hat{\lambda}(\alpha)-\hat{\lambda}\left(\alpha_{0}\right)\right|<\epsilon,\left|\hat{a}(M)-\hat{a}\left(M_{0}\right)\right|<\epsilon\right\} .
$$

If $\nu \in L^{1}$ is such that $\left|\hat{\nu}\left(\alpha_{0}\right)\right|>1$, then, by Lemma 2.2,

$$
N_{1}=\left\{M_{B}:\left|\hat{\nu}\left(\alpha_{B}\right)\right|>1\right\}
$$

is a neighborhood of $M_{B}^{(0)}$. Similarly

$$
\begin{aligned}
& N_{2}=\left\{M_{B}:\left|(a v)^{\wedge}\left(M_{B}\right)-(a \nu)^{\wedge}\left(M_{B}^{(0)}\right)\right|<\epsilon / 2\right\}, \\
& N_{3}=\left\{M_{B}:\left|\hat{\nu}\left(\alpha_{B}\right)-\hat{\nu}\left(\alpha_{0}\right)\right|<\epsilon / 2 \max \left(\left|\hat{a}\left(M_{0}\right)\right|, 1\right)\right\}, \\
& N_{4}=\left\{M_{B}:\left|\hat{\lambda}\left(\alpha_{B}\right)-\hat{\lambda}\left(\alpha_{0}\right)\right|<\epsilon\right\},
\end{aligned}
$$

are neighborhoods of $M_{B}^{(0)}$. Let $N\left(M_{B}^{(0)}\right)$ be the intersection of these four sets. Since $(a \nu)^{\wedge}\left(M_{B}\right)=\hat{a}\left(M^{(B)}\right) \hat{\nu}\left(\alpha_{B}\right)$, it follows, as in the proof of Lemma 2.2, that $N\left(M_{B}^{(0)}\right) \subset N\left(\alpha_{0}, M_{0}\right)$.

Conversely, let

$$
N\left(M_{B}^{(0)}\right)=\left\{M_{B}:\left|\hat{f}\left(M_{B}\right)-\hat{f}\left(M_{B}^{(0)}\right)\right|<\epsilon\right\},
$$

for some $f \in B^{1}$, represent a sub-basis element of the weak topology of $\mathfrak{M}_{B}$. By Lemma 2.3, there exists an open $\hat{G}$-neighborhood $N\left(\alpha_{0}\right)$ of $\alpha_{0}$ such that 
$\alpha \in N\left(\alpha_{0}\right)$ implies $\left\|\int f(x)\left[\bar{\alpha}(x)-\bar{\alpha}_{0}(x)\right] d x\right\|_{\Lambda}<\epsilon / 2$. If

$$
N\left(M_{0}\right)=\left\{M:\left|\int\left[f(x)^{\wedge}(M)-f(x)^{\wedge}\left(M_{0}\right)\right] \bar{\alpha}_{0}(x) d x\right|<\epsilon / 2\right\},
$$

then $N\left(M_{0}\right)$ is open in $\mathfrak{T}_{A}$. Therefore $N\left(\alpha_{0}, M_{0}\right)=N\left(\alpha_{0}\right) \times N\left(M_{0}\right)$ is open in $\hat{G} \times \mathfrak{M}_{A}$ and it contains $\left(\alpha_{0}, M_{0}\right)$. For each $(\alpha, M) \in N\left(\alpha_{0}, M_{0}\right)$ we have

$$
\begin{aligned}
\left|\hat{f}\left(M_{B}^{(\alpha, M)}\right)-\hat{f}\left(M_{B}^{(0)}\right)\right| \leqq & \left|\int f(x)^{\wedge}(M)\left[\bar{\alpha}(x)-\bar{\alpha}_{0}(x)\right] d x\right| \\
& +\left|\int\left[f(x)^{\wedge}(M)-f(x)^{\wedge}\left(M_{0}\right)\right] \bar{\alpha}_{0}(x) d x\right|<\epsilon .
\end{aligned}
$$

Thus $N\left(\alpha_{0}, M_{0}\right) \subset N\left(M_{B}^{(0)}\right)$.

3. Semi-simplicity and the case where $A$ is a group algebra. Some of the properties of $L^{1}$ can be given $B^{1}$ by assuming these properties for $A$. Statements like the next theorem have been proved in the author's thesis for regularity and the Tauberian condition (the set of elements whose Fourier transforms have compact carriers is dense) by extensive use of Theorems 2.6 and 2.7. We shall deal here with semi-simplicity.

Theorem 3.1. $B^{1}$ is semi-simple if and only if $A$ is semi-simple.

Proof. Let $B^{1}$ be semi-simple and let $a \in \bigcap_{9 \pi} M$. Let $\lambda \in L^{1}$ be such that $\|\lambda\|_{1} \neq 0$. By Theorem 2.6, $(a \lambda)^{\wedge}\left(M_{B}\right)=\hat{a}\left(M^{(B)}\right) \hat{\lambda}\left(\alpha_{B}\right)=0$ for every $M_{B} \in \mathscr{T H}_{B}$. Thus $a \lambda \in \cap_{\mathfrak{T T}_{B}} M_{B}=0$ so that $0=\|a \lambda\|_{B}=\|a\|_{A}\|\lambda\|_{1}$, whence $a=0$.

Now suppose $A$ is semi-simple and let $f \in \bigcap_{\mathfrak{T}_{B}} M_{B}$, where $f$ is continuous. Then $\hat{f}\left(M_{B}\right)=0$ for all $M_{B}$ and hence $\int f(x)^{\wedge}(M) \bar{\alpha}(x) d x=0$ for all $\alpha \in \hat{G}$, $M \in \mathfrak{M}_{\boldsymbol{A}} \cdot f(x)^{\wedge}(M)$ is for each $M$ a continuous function in $L^{1}$. Its Fourier transform is identically zero and thus, because $L^{1}$ is semi-simple, $f(x)^{\wedge}(M)=0$ a.e. (almost everywhere). In fact, since it is continuous, $f(x)^{\wedge}(M) \equiv 0$. Hence for each $x, f(x) \in \cap_{\mathfrak{M}}, M=0$, so $f=0$.

We now consider any $f \in \bigcap_{\mathfrak{M T}_{B}} M_{B}$ and assume that $f(x)$ is not a.e. zero. By Theorem 1.3, there exists a uniformly continuous function $u \in L^{1}$ such that $\|u * f\|_{B}>0$. Since each $M_{B}$ is an ideal of $B^{1}(e)$ (see proof of Lemma 2.1), $u * f=u e * f \in M_{B}$ for all $M_{B} \in \mathfrak{M}_{B}$. Furthermore $u * f$ is continuous. This contradicts the preceding paragraph and completes the proof.

We shall now treat the special case where $A$ is a group algebra. Let $A=L^{1}(H)$, where $H$ is a locally compact Abelian group. A function representing an element of $B^{1}\left(G, L^{1}(H)\right)$ takes at each $x \in G$ a value which is an element of $L^{1}(H)$ and this element can be represented by a function over $H$. Thus elements of $B^{1}$ may be thought of as functions over $G \times H$. This double representation of elements of $B^{1}$ does not, however, lead directly to an identification of $B^{1}$ with $L^{1}(G \times H)$. Suppose, for example, that $G$ and $H$ are the reals and let $S$ be Sierpinski's nonmeasurable plane set which intersects 
each line in at most two points [7]. The characteristic function of $S$ represents no element of $L^{1}(G \times H)$, but in the above sense quite properly represents the zero element of $B^{1}\left(G, L^{1}(H)\right)$. It is nevertheless true that the two algebras are isomorphic. The difficulty we have mentioned is easily avoided for elements of a certain dense set and an isomorphism can be established by means of an extension. In the following proof the symbol $\sim$ will be used to distinguish a function from the equivalence class to which it belongs except when the representative is a characteristic function. For any function $\tilde{\lambda}, N(\tilde{\lambda})$ means $\{x: \tilde{\lambda}(x) \neq 0\}$.

Theorem 3.2. If $H$ is a locally compact Abelian group, then $B^{1}\left(G, L^{1}(H)\right)$ is isometric and isomorphic to $L^{1}(G \times H)$.

Proof. Let $B_{0}$ be the subspace of $B^{1}$ generated by elements of the form $\eta \lambda, \eta \in L^{1}(H), \lambda \in L^{1}(G)$. Since $B_{0}$ contains the simple functions, it is dense in $B^{1}$. For $f \in B_{0}, f=\sum_{i=1}^{n} \eta_{i} \lambda_{i}$, we pick representatives $\tilde{\eta}_{i}$ of $\eta_{i}, \tilde{\lambda}_{i}$ of $\lambda_{i}$ and define the function $(T f)$ by the equation

$$
(T f)^{\sim}(x, y)=\sum_{i} \tilde{\eta}_{i}(y) \tilde{\lambda}_{i}(x) .
$$

We shall show that the equivalence class $T f$ to which it belongs is in $L^{1}(G \times H)$.

Let $\tilde{\eta}_{i}^{\prime}(y)=\tilde{\eta}_{i}(y)$ a.e. and $\tilde{\lambda}_{i}^{\prime}(x)=\tilde{\lambda}_{i}(x)$ a.e. Now

$$
\begin{aligned}
\{(x, y): & \left.\tilde{\eta}_{i}(y) \tilde{\lambda}_{i}(x) \neq \tilde{\eta}_{i}^{\prime}(y) \tilde{\lambda}_{i}^{\prime}(x)\right\} \\
& \subset\left[N\left(\tilde{\lambda}_{i}\right) \cup N\left(\tilde{\lambda}_{i}^{\prime}\right)\right] \times N\left(\tilde{\eta}_{i}-\tilde{\eta}_{i}^{\prime}\right) \cup N\left(\tilde{\lambda}_{i}-\tilde{\lambda}_{i}^{\prime}\right) \times\left[N\left(\tilde{\eta}_{i}\right) \cup N\left(\tilde{\eta}_{i}^{\prime}\right)\right]
\end{aligned}
$$

and the larger set has zero measure in $G \times H$. Hence $T f$ is independent of the representatives chosen for the $\eta_{i}$ and $\lambda_{i}$. For $\tilde{\eta}_{i}$ real and $c$ real

$$
\begin{aligned}
\left\{(x, y): \chi_{N\left(\tilde{\lambda}_{i}\right)}(x) \tilde{\eta}_{i}(y) \neq 0\right\} & \cap\left\{(x, y): \chi_{N\left(\tilde{c}_{i}\right)}(x) \tilde{\eta}_{i}(y)<c\right\} \\
& =N\left(\tilde{\lambda}_{i}\right) \times\left[N\left(\tilde{\eta}_{i}\right) \cap\left\{y: \tilde{\eta}_{i}(y)<c\right\}_{J}\right.
\end{aligned}
$$

is measurable. If $\tilde{\eta}_{i}$ is not real, this argument applies to its real and imaginary parts separately. Thus $\chi_{N\left(\tilde{\lambda}_{i}\right)} \tilde{\eta}_{i}$ and, similarly, $\chi_{N\left(\tilde{\eta}_{i}\right)} \tilde{\lambda}_{i}$ are measurable functions, whence $\tilde{\eta}_{i} \tilde{\lambda}_{i}$ is measurable. Consequently for any $f \in B_{0},(T f)^{\sim}$ is measurable and $T f$ is in $L^{1}(G \times H)$; for

$$
\begin{aligned}
\|T f\|_{L^{1}(G \times H)} & =\int_{G} \int_{H}\left|(T f)^{\sim}(x, y)\right| d y d x \\
& =\int_{G}\|\tilde{f}(x)\|_{L^{1}(H)} d x=\|f\|_{B} .
\end{aligned}
$$

$T$ is therefore an isometric linear mapping of the dense subspace $B_{0}$ into $L^{1}(G \times H)$. The image of $B_{0}$ is dense since it contains all $L^{1}$ classes represented by functions of the form $\sum_{i=1}^{n} \sum_{j=1}^{m} c_{i j} \chi_{E_{j}}(y) \chi_{E_{i}}(x)$. Hence $T$ extends uniquely 
to an isometric linear mapping of $B^{1}$ onto $L^{1}(G \times H)$.

It remains to be shown that $T$ preserves convolution. Let $f, g \in B_{0}$, $f=\sum_{i=1}^{n} \eta_{i} \lambda_{i}, g=\sum_{j=1}^{m} \eta_{j}^{\prime} \lambda_{j}^{\prime}$. Then $f * g$ is represented by

$$
(f * g)^{\sim}=\left(\sum_{i} \tilde{\eta}_{i} \tilde{\lambda}_{i}\right) *\left(\sum_{j} \tilde{\eta}_{j}^{\prime} \tilde{\lambda}_{j}^{\prime}\right)=\sum_{i} \sum_{j}\left(\tilde{\eta}_{i} * \tilde{\eta}_{j}^{\prime}\right)\left(\tilde{\lambda}_{i} * \tilde{\lambda}_{j}^{\prime}\right)
$$

and so $f * g \in B_{0}$. Thus

$$
\begin{aligned}
{[T(f * g)]^{\sim}(x, y) } & =\sum_{i} \sum_{j} \int_{H} \tilde{\eta}_{i}(y u) \tilde{\eta}_{j}^{\prime}\left(u^{-1}\right) d u \int_{G} \tilde{\lambda}_{2}(x v) \tilde{\lambda}_{j}^{\prime}\left(v^{-1}\right) d v \\
& =\int_{G} \int_{H} \sum_{i} \tilde{\eta}_{i}(y u) \tilde{\lambda}_{i}(x v) \sum_{j} \tilde{\eta}_{j}^{\prime}\left(u^{-1}\right) \tilde{\lambda}_{j}^{\prime}\left(v^{-1}\right) d u d v \\
& =(T f * T g) \sim(x, y) \text { a.e., }
\end{aligned}
$$

and hence $T(f * g)=T f * T g$. Since convolution is continuous in both its arguments, $T(f * g)=T f * T g$ throughout $B^{1}$.

There is no further need for distinguishing functions from the elements they represent so the customary practice will be resumed.

Note that a maximal ideal of $B^{1}\left(G, L^{1}(H)\right)$ is of the form $\left(\alpha_{1}, \alpha_{2}\right), \alpha_{1} \in \hat{G}$, $\alpha_{2} \in \hat{H}$. For $f \in B_{0}$

$$
\hat{f}\left(\alpha_{1}, \alpha_{2}\right)=\int_{G} f(x)^{\wedge}\left(\alpha_{2}\right) \bar{\alpha}_{1}(x) d x=\int_{G} \int_{H}(T f)(x, y) \bar{\alpha}_{2}(y) \bar{\alpha}_{1}(x) d y d x,
$$

which is the integral form of the Fourier transform of $T f \in L^{1}(G \times H)$ at $\left(\alpha_{1}, \alpha_{2}\right) \in \hat{G} \times \hat{H}$. The fact that $\hat{f}\left(\alpha_{1}, \alpha_{2}\right)=(T f)^{\wedge}\left(\alpha_{1}, \alpha_{2}\right)$ for all $f \in B^{1}$ follows immediately.

4. Isomorphisms of Banach algebras of the form $B^{1}$. An isomorphism between two topological groups or Banach algebras will mean an algebraic isomorphism which is also a homeomorphism. $G$ and $\tilde{G}$ will be locally compact Abelian groups and $A$ and $\tilde{A}$ commutative Banach algebras. If $G$ is isomorphic to $\widetilde{G}$ and $A$ is isomorphic to $\tilde{A}$, isomorphisms of $B^{1}(G, A)$ and $B^{1}(\widetilde{G}, \tilde{A})$ can be easily constructed (Theorem 4.1 ); but the determination of conditions under which the converse is true is, as we shall see, more difficult.

THEOREM 4.1. Let $\tau$ be an isomorphism of $G$ onto $\tilde{G}$ and $J$ an isomorphism of $A$ onto $\tilde{A}$. Let $k$ denote the constant determined by $d x=k d \tau x$. Then for any $\alpha \in \hat{G}$, the mapping $T$ defined by the equation

$$
(T f)(\tau x)=k \alpha(x) \Im[f(x)]
$$

is an isomorphism of $B^{1}(G, A)$ onto $B^{1}(\widetilde{G}, \widetilde{A}) . T$ is an isometry if and only if $J$ is an isometry.

Proof. If $f \in B^{1}(G, A), T f$ is measurable and 


$$
\int\|(T f)(\tau x)\|_{\tilde{A}} d \tau x=\int\|\Im[f(x)]\| \tilde{A} d x \leqq\|\Im\|\|f\|_{B} .
$$

Thus $T f \in B^{1}(\tilde{G}, \tilde{A})$ and, since $T$ is clearly linear, it is continuous. If $\tilde{f} \in B^{1}(\tilde{G}, \tilde{A})$, then $f\left(\tau^{-1} \tilde{x}\right)=k^{-1} \bar{\alpha}\left(\tau^{-1} \tilde{x}\right) J^{-1}[\tilde{f}(\tilde{x})]$ represents its unique preimage and $\|f\|_{B} \leqq\left\|\mathcal{J}^{-1}\right\|\|\tilde{f}\|_{\tilde{B}}$. $T$ is therefore one-to-one, onto, and bicontinuous. Finally, for $f, g \in B^{1}(G, A)$

$$
\begin{aligned}
(T(f * g))(\tau x) & =k \alpha(x) J \int f(x y) g\left(y^{-1}\right) d y \\
& =\int(T f)(\tau x \tau y)(T g)\left[(\tau y)^{-1}\right] d \tau y=(T f * T g)(\tau x) .
\end{aligned}
$$

$T$ is clearly an isometry if $J$ is. Conversely, if $T$ is an isometry, for any $a \in A$ and $\lambda \in L^{1}(G)$ where $\|\lambda\|_{1}=1$, we have $(T(a \lambda))(\tau x)=k \alpha(x) \lambda(x) J a$ and

$$
\|a\|_{A}=\|a \lambda\|_{B}=\|T(a \lambda)\|_{\tilde{B}}=\|\Im a\|_{\tilde{A}} .
$$

In the special case of group algebras both $A$ and $\tilde{A}$ are the complex numbers, $J$ is necessarily the identity transformation, and the converse of this theorem holds provided $T$ is an isometry. The following result is due to Wendel [8].

If $T$ is an isometric isomorphism of $L^{1}(G)$ onto $L^{1}(\tilde{G})$, there exist an isomorphism $\tau$ of $G$ onto $\tilde{G}$ and a character $\alpha \in \tilde{G}$ such that for every $\lambda \in L^{1}(G)$, $T \lambda_{x}=\bar{\alpha} \cdot(T \lambda)_{\tau x}$ and $(T \lambda)(\tau x)=k \alpha(x) \lambda(x)$, where $k$ is such that $d x=k d \tau x$.

It suffices to assume that $T$ is an isomorphism such that $\|T\| \leqq 1$ (see Helson [1]), in which case $T$ must again be an isometry. However, isomorphism alone is not sufficient for there are nonisomorphic groups whose algebras are isomorphic [8]. Algebras of the form $B^{1}$ display even greater pathology since an appeal to isometry fails to produce isomorphisms of the component spaces. Indeed, we know that $B^{1}\left(G, L^{1}(H)\right)$ is isometric and isomorphic to $L^{1}(G \times H)=B^{1}(G \times H, C), C$ being the complexes (Theorem 3.2), but in general the corresponding components are not isomorphic.

It is not even true that isomorphism between just one pair of the components can be forced by assuming isomorphism between the other pair along with an isometric isomorphism of $B^{1}(G, A)$ on to $B(\tilde{G}, \tilde{A})$. We proceed to counterexamples.

Consider an infinite-dimensional Hilbert space $\mathcal{F} . \mathcal{H}$ is isomorphic to the direct sum $\mathfrak{K} \times \mathfrak{K C}$, and the additive group $H$ of $\mathfrak{H C}$ becomes locally compact when assigned the discrete topology. $H$ and $H \times H$ are algebraically isomorphic by virtue of the Hilbert space isomorphism and homeomorphic because they are discrete spaces in one-to-one correspondence. $B^{1}\left(H, L^{1}(H)\right)$ is then isometrically isomorphic to $L^{1}(H \times H)$ and therefore to $L^{1}(H)$. This is also true for the compact group $\hat{H}$ since it is isomorphic to $\hat{H} \times \hat{H}$. Hence even 
for compact groups $G, B^{1}(G, A)$ isomorphic to $L^{1}(G)$ does not imply that $A$ is the complex numbers.

In regard to the case in which $A$ is isomorphic to $\tilde{A}$, we note that for any $G$ and $H, B^{1}\left(G \times H, L^{1}(H)\right)$ is isometric and isomorphic to $L^{1}(G \times H \times H)$ and hence to $B^{1}\left(G, L^{1}(H \times H)\right)$. The counterexample comes from taking $H$ isomorphic to $H \times H$.

It is therefore by no means the case that every isomorphism $T$ is of the triple form $(J, \tau, \alpha)$. However

THeOReM 4.2. If the triples $\left(J_{1}, \tau_{1}, \alpha_{1}\right)$ and $\left(J_{2}, \tau_{2}, \alpha_{2}\right)$ are distinct, the corresponding isomorphisms $T_{1}$ and $T_{2}$ are distinct.

Proof. We treat four cases. Subscripts are not written on corresponding components assumed to be equal.

CASE 1. $\tau_{1} \neq \tau_{2}$. Let $\tilde{x} \in \tilde{G}$ be such that $\tau_{1}^{-1} \tilde{x} \neq \tau_{2}^{-1} \tilde{x}$. Choose disjoint open neighborhoods $U, V$ of $\tau_{1}^{-1} \tilde{x}, \tau_{2}^{-1} \tilde{x}$ such that $\mu(U)<\infty, \mu(V)<\infty$ and let $f \in B^{1}(G, A)$ be zero on $U$ but not on $V$. Then $T_{1} f \neq T_{2} f$ on $\tau_{1} U \cap \tau_{2} V$, a set of positive measure.

CASE 2. $\Im_{1} \neq J_{2}, \tau_{1}=\tau_{2}, \alpha_{1}=\alpha_{2}$. If $a \in A$ is such that $\Im_{1}(a) \neq J_{2}(a)$ and $f=a$ on a set $E$ of finite nonzero measure, then $T_{1} f \neq T_{2} f$ on $\tau E$.

CASE 3. $\alpha_{1} \neq \alpha_{2}, J_{1}=J_{2}, \tau_{1}=\tau_{2}$. Let $E_{1}$ be a set of finite nonzero measure contained in the open set $E=\left\{x: \alpha_{1}(x) \neq \alpha_{2}(x)\right\}$. If $f=a \neq 0$ on $E_{1}, T_{1} f \neq T_{2} f$ on $\tau E_{1}$ since $k \alpha_{1}(x) \mathfrak{J}(a)=k \alpha_{2}(x) \mathfrak{J}(a)$ implies $x \in E$.

CASE 4. $J_{1} \neq J_{2}, \alpha_{1} \neq \alpha_{2}, \tau_{1}=\tau_{2}$. Let $a$ be such that $\Im_{1}(a) \neq J_{2}(a)$ and let $E=\left\{x: \alpha_{1}(x) \neq \alpha_{2}(x)\right\}$. If $J_{1}(a)$ is not a scalar multiple of $J_{2}(a)$, the case is settled by $f=a$ on a set of finite nonzero measure in $E$. Suppose that $\Im_{1}(a)$ $=c J_{2}(a)$, where $c \neq 1$ is complex. Either there exists $E_{1} \subset E$ of finite nonzero measure on which $\alpha_{1} \neq c \alpha_{2}$ or $E=\left\{x: \alpha_{1} \alpha_{2}^{-1}(x)=c\right\}$. In the first instance choose $f=a$ on $E_{1}$. In the second $E^{\prime}$ is open and nonempty since it contains $e$. Let $E_{2} \subset E^{\prime}$ be of finite nonzero measure and take $f=a$ on $E_{2}$.

The purpose of the remainder of this paper is to obtain conditions under which an isomorphism $T$ must be of the form $(\mathcal{J}, \tau, \alpha)$. If $M_{B}$ is a maximal ideal of $B^{1}(G, A), T M_{B}$ is a maximal ideal of $B^{1}(\tilde{G}, \tilde{A})$. Also $\hat{f}\left(M_{B}\right)$ $=(T f)^{\wedge}\left(T M_{B}\right), f \in B^{1}(G, A)$, for the kernel of the homomorphism $f \rightarrow(T f)^{\wedge}\left(T M_{B}\right)$ is clearly $M_{B}$. Since $M_{B}$ is of the form $(\alpha, M), \alpha \in \hat{G}, M \in \mathfrak{M}_{A}$, and $T M_{B}$ is of the form $(\tilde{\alpha}, \tilde{M}), \tilde{\alpha} \in \tilde{G}^{\wedge}, \tilde{M} \in \mathfrak{M}_{\tilde{A}}, T$ gives rise to correspondences between the characters of $G$ and $\tilde{G}$ and the maximal ideals of $A$ and $\tilde{A}$. However, these correspondences can be intractable. Consider, for example, the isomorphism $T$ of $B^{1}\left(G, L^{1}(H)\right)$ onto $L^{1}(G \times H)$. Since $\hat{f}\left(\alpha_{1}, \alpha_{2}\right)$ $=(T f)^{\wedge}\left(\alpha_{1}, \alpha_{2}\right), \alpha_{1} \in \hat{G}, \alpha_{2} \in \hat{H}, f \in B^{1}$ (see remarks at end of \$3), $T\left(\alpha_{1}, \alpha_{2}\right)$ $=\left(\left(\alpha_{1}, \alpha_{2}\right),(0)\right)$, where $(0)$ is the single ideal of the complexes. If $\alpha_{1}$ is held fixed and $\alpha_{2}$ is varied, the maximal ideal component of $T\left(\alpha_{1}, \alpha_{2}\right)$ cannot change but the character component does. To allow for this kind of behavior we shall use the notation $T(\alpha, M)=\left(\tilde{\alpha}_{\alpha, M}, \tilde{M}_{\alpha, M}\right)$. 
If $T$ is a triple $\left(\mathcal{J}, \tau, \alpha_{0}\right)$, the complications discussed in the last paragraph do not appear. For any $s \in G, f \in B^{1}(G, A)$,

$$
\left(T f_{8}\right)(\tau x)=k \alpha_{0}(x) \Im\left[f_{8}(x)\right]=\bar{\alpha}_{0}(s)(T f)(\tau s x)=\bar{\alpha}_{0}(s)(T f)_{\tau \delta}(\tau x) .
$$

Hence, if $f \notin M_{B}=(\alpha, M)$, by Theorem 2.3,

$$
\begin{aligned}
\tilde{\alpha}_{\alpha, M}(\tau x) & =\frac{(T f)_{\tau x}\left(T M_{B}\right)}{(T f)^{\wedge}\left(T M_{B}\right)}=\frac{\alpha_{0}(x)\left(T f_{x}\right)^{\wedge}\left(T M_{B}\right)}{(T f)^{\wedge}\left(T M_{B}\right)}=\frac{\alpha_{0}(x) \hat{f}_{x}\left(M_{B}\right)}{\hat{f}\left(M_{B}\right)} \\
& =\alpha_{0}(x) \alpha(x) .
\end{aligned}
$$

If $\lambda$ is such that $\lambda(\alpha)=1$, then for any $a \in A$ the integral formula yields

$$
\begin{aligned}
\hat{a}(M) & =(a \lambda)^{\wedge}\left(M_{B}\right)=[T(a \lambda)]^{\wedge}\left(T M_{B}\right) \\
& =\int k \alpha_{0}(x)(\Im[a \lambda(x)])^{\wedge}\left(\tilde{M}_{\alpha, M}\right) \tilde{\alpha}_{\alpha, M}^{-}(\tau x) d \tau x \\
& =(J a)^{\wedge}\left(\tilde{M}_{\alpha, M}\right) \int \alpha_{0}(x) \lambda(x) \bar{\alpha}_{0}(x) \bar{\alpha}(x) d x=(J a)^{\wedge}\left(\tilde{M}_{\alpha, M}\right) .
\end{aligned}
$$

Hence for every $M \in \mathfrak{M T}_{A}, \tilde{M}_{\alpha, M}=\Im M$ regardless of $\alpha$ and for every $\alpha \in \hat{G}$, $\tilde{\alpha}_{\alpha, M}(\tau x)=\alpha_{0}(x) \alpha(x)$ regardless of $M$. Conversely, if $B^{1}(\tilde{G}, \tilde{A})$ is semi-simple, these conditions guarantee that $T$ is the triple $\left(J, \tau, \alpha_{0}\right)$. Before proving this we observe that the semi-simplicity of any one of the algebras $A, \widetilde{A}, B^{1}(G, A)$, or $B^{1}(\tilde{G}, \tilde{A})$ implies that of the others (Theorem 3.1). In the statements of results requiring semi-simplicity we shall say merely that $A$ is semi-simple.

Lemma 4.1. Let $T$ be an isomorphism of $B^{1}(G, A)$ onto $B^{1}(\tilde{G}, \widetilde{A})$, where $A$ is semi-simple. Let $\mathrm{J}$ be an isomorphism of $A$ onto $\tilde{A}, \tau$ an isomorphism of $G$ onto $\tilde{G}$, and $\alpha_{0} \in \hat{G}$ such that for every $\alpha \in \hat{G}, M \in \mathfrak{N}_{A}, \tilde{\alpha}_{\alpha, M}(\tau x)=\alpha_{0}(x) \alpha(x)$ and $\tilde{M}_{\alpha, M}=\Im M$. Then $T$ is the isomorphism (J, $\left.\tau, \alpha_{0}\right)$.

Proof. Let $T_{1}$ denote the isomorphism $\left(\mathcal{J}, \tau, \alpha_{0}\right)$. If $f \in B^{1}(G, A)$, for any $M_{B}=(\alpha, M)$

$$
\begin{aligned}
\left(T_{1} f\right)^{\wedge}\left(T M_{B}\right) & =\int \alpha_{0}(x)(\Im[f(x)])^{\wedge}(J M) \bar{\alpha}_{0}(x) \bar{\alpha}(x) d x \\
& =\int f(x)^{\wedge}(M) \bar{\alpha}(x) d x=\hat{f}\left(M_{B}\right)=(T f)^{\wedge}\left(T M_{B}\right) .
\end{aligned}
$$

Since every maximal ideal of the semi-simple algebra $B^{1}(\tilde{G}, \tilde{A})$ has the form $T M_{B}, T_{1} f=T f$ for every $f \in B^{1}(G, A)$.

Let us examine further the case where $T$ is given as (J, $\left.\tau, \alpha_{0}\right)$. Let $\left(\alpha_{1}, M_{1}\right)$ be a fixed but arbitrary maximal ideal of $B^{1}(G, A)$. Then $f \in \cap_{\mathscr{F}_{A}}\left(\alpha_{1}, M\right)$ if and only if $\int f(x) \bar{\alpha}_{1}(x) d x \in \cap_{\mathfrak{F}_{A}} M$. Since 


$$
\int(T f)(\tau x) \tilde{\alpha}_{\alpha_{1}, M_{1}}(\tau x) d \tau x=\mathfrak{J} \int f(x) \bar{\alpha}_{1}(x) d x,
$$

this is equivalent to

$$
\int(T f)(\tau x) \tilde{\alpha}_{\alpha_{1}, M_{1}}^{-}(\tau x) d \tau x \in \cap_{\mathfrak{M}_{A}} \mathfrak{J} M=\cap_{\mathfrak{N}_{\tilde{A}} \tilde{M}}
$$

and hence to $T f \in \bigcap_{\mathscr{M}_{\tilde{A}}}\left(\tilde{\alpha}_{\alpha_{1}, M_{1}}, \tilde{M}\right)$. Thus

$$
T\left[\cap_{\mathscr{T}_{A}}\left(\alpha_{1}, M\right)\right]=\bigcap_{\mathscr{T}_{\tilde{A}}}\left(\tilde{\alpha}_{\alpha_{1}, M_{1}}, \tilde{M}\right) .
$$

Also $f \in \bigcap_{\hat{G}}\left(\alpha, M_{1}\right)$ if and only if $f(x) \in M_{1}$ for almost every $x$, which is true if and only if $(T f)(\tau x) \in J M_{1}=\tilde{M}_{\alpha_{1}, M_{1}}$ for almost every $\tau x$. Since the last is equivalent to $T f \in \bigcap_{\tilde{G} \wedge}\left(\tilde{a}, M_{\alpha_{1}, M_{1}}\right)$,

$$
T\left[\cap_{\tilde{G}}\left(\alpha, M_{1}\right)\right]=\bigcap_{\tilde{G}^{-}}\left(\tilde{\alpha}, \tilde{M}_{\alpha_{1}, M_{1}}\right) .
$$

These are essentially the conditions which will be used to obtain a statement converse to Theorem 4.1. Their intrinsic appeal lies in the fact that they are basic requirements for the construction from $T$ of a $J$ and an isomorphism of $L^{1}(G)$ onto $L^{1}(\tilde{G})$ by means of the Fourier transform. Note that these conditions do not hold for our isomorphism of $B^{1}\left(G, L^{1}(H)\right)$ onto $L^{1}(G \times H)$.

Definition. Let $T$ be an isomorphism of $B^{1}(G, A)$ onto $B^{1}(\tilde{G}, \tilde{A})$. Condition $I$ is said to hold for $T$ at $\alpha_{1} \in \hat{G}$ if there exists $M_{1} \in \mathfrak{M}_{A}$ such that $T\left[\cap_{\mathscr{T H}_{\boldsymbol{A}}}\left(\alpha_{1}, M\right)\right]=\bigcap_{\mathfrak{T}_{\tilde{\boldsymbol{A}}}}\left(\tilde{\alpha}_{\alpha_{1}, M_{1}}, \tilde{M}\right)$. Condition II is said to hold for $T$ at $M_{1} \in \mathfrak{M}_{A}$ if there exists $\alpha_{1} \in \hat{G}$ such that $T\left[\bigcap_{\widehat{G}}\left(\alpha, M_{1}\right)\right]=\bigcap_{\tilde{G}^{\wedge}}\left(\tilde{\alpha}, \tilde{M}_{\alpha_{1}, M_{1}}\right)$.

Lemma 4.2. Let $A$ be semi-simple. If $T$ is an isomorphism for which Condition I holds at some $\alpha_{1} \in \hat{G}$, then there is an isomorphism $\Im_{\alpha_{1}}$ of $A$ onto $\tilde{A}$, and for every $M \in \mathfrak{M}_{A}$

$$
\tilde{\alpha}_{\alpha_{1}, M}=\tilde{\alpha}_{\alpha_{1}, M_{1}}, \quad \tilde{M}_{\alpha_{1}, M}=J_{\alpha_{1}} M .
$$

Proof. Suppose that there exists $M_{2} \in 9 \pi_{A}$ such that $\tilde{\alpha}_{\alpha_{1}, M_{2}} \neq \tilde{\alpha}_{\alpha_{1}, M_{1}}$. Choose $\tilde{\lambda} \in L^{1}(\tilde{G})$ such that $\tilde{\lambda}^{\wedge}\left(\tilde{\alpha}_{\alpha_{1}, M_{1}}\right)=0$ and $\tilde{\lambda}^{\wedge}\left(\tilde{\alpha}_{\alpha_{1}, M_{2}}\right) \neq 0$. If $\tilde{a} \notin \bar{M}_{\alpha_{1}, M_{2}}$, then $\tilde{a} \tilde{\lambda} \notin\left(\tilde{\alpha}_{\alpha_{1}, M_{2}}\right) \tilde{M}_{\alpha_{1}, M_{2}}=T\left(\alpha_{1}, M_{2}\right)$. But $\tilde{a} \tilde{\lambda} \in \bigcap_{\mathfrak{T}_{\tilde{A}}}\left(\tilde{\alpha}_{\alpha_{1}, M_{1}}, \tilde{M}\right)$ and hence, by Condition I, $T^{-1}(\tilde{a} \tilde{\lambda}) \in \bigcap_{\mathfrak{T}_{A}}\left(\alpha_{1}, M\right)$. In particular $T^{-1}(\tilde{a} \tilde{\lambda}) \in\left(\alpha_{1}, M_{2}\right)$ so that $\tilde{a} \tilde{\lambda}$ $\in T\left(\alpha_{1}, M_{2}\right)$, which is impossible. Therefore $\tilde{\alpha}_{\alpha_{1}, M}=\tilde{\alpha}_{\alpha_{1}, M}$ for every $M \in \mathbb{T}_{A}$.

Theorem 2.1 asserts that for any $a \in A$ there exists $f \in B^{1}(G, A)$ such that $\int f(x) \bar{\alpha}_{1}(x) d x=a$, and we accordingly set

$$
\Im_{\alpha_{1}} a=\int(T f)(\tilde{x}) \tilde{\alpha}_{\alpha_{1}, M_{1}}(\tilde{x}) d \tilde{x} .
$$


If $g$ is such that $\int g(x) \bar{\alpha}_{1}(x) d x=a$, then, by Condition I, $T f-T g$ $\in \bigcap_{\mathfrak{N}_{\tilde{\Lambda}}}\left(\tilde{\alpha}_{\alpha_{1}, M_{1}}, \tilde{M}\right)$, and since $\tilde{A}$ is semi-simple,

$$
\int(T f)(\tilde{x}) \tilde{\alpha}_{\alpha_{1}, M_{1}}^{-}(\tilde{x}) d \tilde{x}=\int(T g)(\tilde{x}) \tilde{\alpha}_{\alpha_{1}, M_{1}}(\tilde{x}) d \tilde{x} .
$$

Thus $\Im_{\alpha_{1}}$ is a well defined mapping of $A$ into $\tilde{A}$. Because of the symmetry of Condition I and the semi-simplicity of $A$, the argument just used can be reversed, and therefore $J_{\alpha_{1}}$ is one-to-one. It follows directly from Theorem 2.1 and the fact that $T$ is an isomorphism that $J_{\alpha_{1}}$ maps $A$ onto $\tilde{A}$ and that it is linear and multiplicative. Since the topology of a commutative semi-simple Banach algebra is uniquely determined, $J_{\alpha_{1}}$ is, in addition, a homeomorphism.

If $f$ is such that $\int f(x) \bar{\alpha}_{1}(x) d x=a$, the following four conditions are equivalent: $a \in M ; f \in\left(\alpha_{1}, M\right) ; T f \in\left(\tilde{\alpha}_{\alpha_{1}, M}, \tilde{M}_{\alpha_{1}, M}\right)\left(=\left(\tilde{\alpha}_{\alpha_{1}, M_{1}}, \tilde{M}_{\alpha_{1}, M}\right)\right) ; \Im_{\alpha_{1}} a \in \tilde{M}_{\alpha_{1}, M}$. Hence $\widetilde{M}_{\alpha_{1}, M}=J_{\alpha_{1}} M$ for every $M \in \mathscr{T}_{A}$.

If $S$ is an isomorphism of $L^{1}(G)$ onto $L^{1}(\tilde{G})$ and $\alpha \in \hat{G}$, the maximal ideal to which $\alpha$ corresponds is carried by $S$ into a maximal ideal of $L^{1}(\widetilde{G})$. The corresponding character will be denoted by $S \alpha$. The next result parallels the last and provides a means for constructing the group isomorphism we seek. This time semi-simplicity is not required since our concern is with group algebras.

Lemma 4.3. If $T$ is an isomorphism for which Condition II holds at some $M_{1} \in \mathfrak{M}_{A}$, then there is an isomorphism $S_{M_{1}}$ of $L^{1}(G)$ onto $L^{1}(\tilde{G})$, and for every $\alpha \in \hat{G}$

$$
\tilde{M}_{\alpha, M_{1}}=\tilde{M}_{\alpha_{1}, M_{1}}, \quad \tilde{\alpha}_{\alpha, M_{1}}=S_{M_{1} \alpha} .
$$

Proof. Because of the obvious similarity to Lemma 4.2, we shall carry through only the construction of $S_{M_{1}}$. If $f \in B^{1}(G, A)$, the function $\lambda$ determined by $\lambda(x)=f(x)^{\wedge}\left(M_{1}\right)$ is in $L^{1}(G)$. A linear mapping of $B^{1}(G, A)$ onto $L^{1}(G)$ is thereby defined by $M_{1}$. The mapping is also a homomorphism; for if $f(x)^{\wedge}\left(M_{1}\right)=\lambda(x)$ a.e. and $g(x)^{\wedge}\left(M_{1}\right)=\nu(x)$ a.e., then

$$
(f * g)(x)^{\wedge}\left(M_{1}\right)=\int f(x y)^{\wedge}\left(M_{1}\right) g\left(y^{-1}\right)\left(M_{1}\right) d y=(\lambda * \nu)(x) \text { a.e. }
$$

$\widetilde{M}_{\alpha_{1}, M_{1}}$ provides in like manner a homomorphism of $B^{1}(\widetilde{G}, \tilde{A})$ onto $L^{\prime}(\widetilde{G})$. Let $S_{M_{1}} \lambda$ be defined by the equation

$$
\left(S_{M_{1}} \lambda\right)(\tilde{x})=(T f)(\tilde{x})^{\wedge}\left(\tilde{M}_{\alpha_{1}, M_{1}}\right),
$$

where $f$ is such that $f(x)^{\wedge}\left(M_{1}\right)=\lambda(x)$ a.e. Arguments much like those used above complete the proof.

If $T$ is an isometry, or even such that $\|T\| \leqq 1$, a technique which will be used in the proof of the next theorem shows that $S_{M_{1}}$ is an isometry. Wendel's theorem then produces an isomorphism of the groups and a character. A 
question which has not been answered is whether these assumptions combined with those of Lemma 4.2 are sufficient to make $T$ a triple. We shall instead assume that Conditions I and II hold everywhere.

THEOREM 4.3. Let $A$ be semi-simple and $T$ an isometric isomorphism of $B^{1}(G, A)$ onto $B^{1}(\tilde{G}, \tilde{A})$ for which Condition I holds at every $\alpha \in \hat{G}$ and Condition II holds at every $M \in \mathfrak{M}_{A}$. Then there exist an isometric isomorphism $\exists$ of $A$ onto $\tilde{A}$, an isomorphism $\tau$ of $G$ onto $\widetilde{G}$, and a character $\alpha_{0} \in \hat{G}$ such that $T$ is the isomorphism (J, $\left.\tau, \alpha_{0}\right)$.

Proof. Let $\alpha_{1}$ and $\alpha_{2}$ be arbitrary characters of $G$. Since the hypotheses of Lemma 4.2 are satisfied for both characters, there exist isomorphisms $J_{\alpha_{1}}$ and $J_{\alpha_{2}}$ of $A$ onto $\tilde{A}$ such that for every $M \in \mathscr{M}_{A}, J_{\alpha_{1}} M=\tilde{M}_{\alpha_{1}, M}$ and $\Im_{\alpha_{2}} M=\tilde{M}_{\alpha_{2}, M}$. Since Lemma 4.3 obtains for every $M, \tilde{M}_{\alpha_{1}, M}=\tilde{M}_{\alpha_{2}, M}$. Hence $J_{\alpha_{1}} M=J_{\alpha_{2}} M$

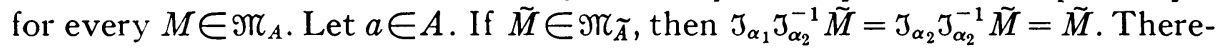
fore

$$
\left(J_{\alpha_{1}} a\right)^{\wedge}(\tilde{M})=\left(J_{\alpha_{1}} a\right)^{\wedge}\left(J_{\alpha_{1}} J_{\alpha_{2}}^{-1} \tilde{M}\right)=\hat{a}\left(J_{\alpha_{2}}^{-1} \tilde{M}\right)=\left(J_{\alpha_{2}} a\right)^{\wedge}(\tilde{M}) .
$$

Thus for every $a \in A,\left(J_{\alpha_{1}} a\right)^{\wedge} \equiv\left(J_{\alpha_{2}} a\right)^{\wedge}$ and it follows from the semi-simplicity of $\tilde{A}$ that $J_{\alpha_{1}} a=J_{\alpha_{2}} a$. Hence $J_{\alpha_{1}}$ and $J_{\alpha_{2}}$ are identical isomorphisms. Since $\alpha_{1}$ and $\alpha_{2}$ are arbitrary, $J \equiv J_{\alpha}$, for any $\alpha$, is independent of $\alpha$, and $J M=\tilde{M}_{\alpha, M}$ for every $\alpha \in \hat{G}, M \in \mathfrak{M}_{A}$.

Let $S_{M}, M \in \mathfrak{M}_{A}$, be the isomorphism of $L^{1}(G)$ onto $L^{1}(\tilde{G})$ given by Lemma 4.3. In the fashion of the preceding paragraph we deduce that $S=S_{M}$ is independent of $M$, and $S \alpha=\tilde{\alpha}_{\alpha, M}$ for every $\alpha \in \hat{G}, M \in \mathfrak{M}_{\Lambda}$.

If $M \in \mathscr{M}_{A}$ and $u_{0}$ is an identity modulo $M$, then

$$
1=\hat{u}_{0}(M)=\left|\hat{u}_{0}(M)\right|=\inf _{u \in u_{0}+M}\|u\|_{A} .
$$

Hence for $\epsilon>0$, there exists an identity $u_{1}$ modulo $M$ such that $\left\|u_{1}\right\|_{A}<1+\epsilon$. For $\lambda \in L^{\mathfrak{1}}(G)$ and $f=u_{1} \lambda$, we have $f(x)^{\wedge}(M)=\lambda(x)$. By the definition of $S_{M}$ (see proof of Lemma 4.3) and because $T$ is an isometry,

$$
\begin{aligned}
\left\|S_{M} \lambda\right\|_{L^{1}(\tilde{G})} & \leqq\|T f\|_{\tilde{B}}=\|f\|_{B}=\left\|u_{1}\right\|_{A}\|\lambda\|_{L^{1}(G)} \\
& <(1+\epsilon)\|\lambda\|_{L^{1}(G) .}
\end{aligned}
$$

Since $\epsilon$ is arbitrary, $\left\|S_{M} \lambda\right\|_{L^{1}(\tilde{G})} \leqq\|\lambda\|_{L^{1}(G)}$. Thus $\|S\| \equiv\left\|S_{M}\right\| \leqq 1$. Wendel's theorem then applies. There exist an isomorphism $\tau$ of $G$ onto $\tilde{G}$ and a character $\alpha_{0}$ such that for every $\lambda \in L^{1}(G)$ and $x \in G, S \lambda_{x}=\bar{\alpha}_{0} \cdot(S \lambda)_{\tau x}$. Let $\alpha \in \hat{G}$. If $\lambda(\alpha) \neq 0$, then

$$
\begin{aligned}
\tilde{\alpha}_{\alpha, M}(\tau x)=(S \alpha)(\tau x) & =\frac{(S \lambda)_{\tilde{\tau}}(S \alpha)}{(S \lambda)^{\wedge}(S \alpha)}=\alpha_{0}(x) \frac{\left(S \lambda_{x}\right)^{\wedge}(S \alpha)}{(S \lambda)^{\wedge}(S \alpha)} \\
& =\alpha_{0}(x) \frac{\hat{\lambda}_{x}(\alpha)}{\hat{\lambda}(\alpha)}=\alpha_{0}(x) \alpha(x)
\end{aligned}
$$


for every $M \in \mathfrak{M}_{A}$. It follows from Lemma 4.1 that $T=\left(\mathcal{T}, \tau, \alpha_{0}\right)$ and, therefore, from Theorem 4.1 , that $J$ is an isometry.

\section{BIBLIOGRAPHY}

1. H. Helson, Isomorphisms of abelian group algebras, Ark. Mat. vol. 2 (1954) pp. 475-487.

2. E. Hille, Functional analysis and semi-groups, Amer. Math. Soc. Colloquium Publications, vol. 31, New York, 1948.

3. L. H. Loomis, An introduction to abstract harmonic analysis, New York, 1953.

4. J. von Neumann, Almost periodic functions in a group, Trans. Amer. Math. Soc. vol. 36 (1934) pp. 445-492.

5. D. A. Raikov, Harmonic analysis on commutative groups with the Haar measure and the theory of characters, Trav. Inst. Math. Stekloff vol. 14 (1945).

6. I. E. Segal, The group algebra of a locally compact group, Trans. Amer. Math. Soc. vol. 61 (1947) pp. 69-105.

7. W. Sierpinski, Sur un problème concernant les ensembles measurables superficiellement, Fund. Math. vol. 1 (1920) pp. 112-115.

8. J. Wendel, On isometric isomorphism of group algebras, Pacific J. Math. vol. 1 (1951) pp. $305-311$.

University of Minnesota, MinNEAPOLIS, MinNESOTA 